\title{
Evidence Based Nurses' Practice for Children Undergoing Abdominal Stoma Outcome
}

Doaa R Maohamed , master degree of pediatric nursing

faculty of nursing, Tanta university

Ebtisam M El Sayed, prof of pediatric nursing

faculty of nursing, Tanta university

Hamada H Husain , Professor of Pediatric Surgery

Faculty of Medicine Tanta University

Nagafa H Farag, Lecturer of Pediatric Nursing

Faculty of Nursing, Tanta University

\begin{abstract}
Aim: This study aimed to evaluate evidence based nurses' practice for children undergoing abdominal stoma outcome. Materials and Method: A quasi-experimental design was utilized in this study, that was conducted in Pediatric Surgical Department of Tanta University Hospital. All available nurses (40 nurses) working in previously mentioned setting and a purposive sample of 40 children divided into two equal groups and admitted to pediatric surgical unit undergoing abdominal stoma operation were taken from the above mentioned setting. Structured questionnaire schedule to assess socio demographic data of nurses and children, Nurses' knowledge regarding to stoma care 2) Nurses performance checklist regarding stoma care, peristomal skin observation checklist to observe the quality of nursing care provided for children undergoing stoma operation and detect the extent and severity of stoma and peristomal skin complications. Results : most of the nurses had poor levels of knowledge and performance related to stoma operation before application of evidence based practice and their knowledge and performance improved immediately and post three months of evidence based practice Moreover, conditions of stomas and peristomal skin improved after nurses practiced evidence based practice. Conclusion : On the light of the current study results, it can be concluded that educational and training program was effective regarding improvement of nurses' knowledge and performance. Recommendations: continuing periodically in-service training programs should be introduced to the nurses on regular basis by different methods and materials of teaching .
\end{abstract}

Keywords : evidence based practice, abdominal stomas, outcomes 


\section{Introduction}

Stoma is surgically created opening that connects a portion of a body cavity to the external environment. Parts of the gastrointestinal tract (GIT) which could be involved in the creation of a stoma are the esophagus, stomach, duodenum, small bowel and colon. The visible portion of the ostomy is called a stoma. (1) The most common indications for stoma surgery are inflammatory bowel diseases (ulcerative colitis or Crohn's disease ) and cancer that interfere with gastrointestinal or genitourinary tract infection. Children with congenital disorders such as hirschsprung's disease and spina bifida, imperforate anus, meconium ileus, necrotizing enterocolitis, and pseudomembranous enterocolitis. In some cases, stoma bypasses trauma site to allow necessary time to heal. Stoma can be permanent, but in the majority, almost two thirds, of children who require a stoma do so on temporary basis. There are two peaks for formation of stoma in the neonatal period and the teenage years (2) .There are several types of stomas performed in the abdominal wall, some are temporary and the others are permanent. Stomas from the colon are named by the part of the colon in which stomas are constructed such as sigmoid, descending, and transverse, colon stomas are called colostomies . Stomas from small intestine are called jejunostoma, or ileostoma. Stoma performed to drainage of urine called urostomies, stoma performed to feed the patient through the stomach called gastrostomy ${ }^{(3)}$.In Egypt during a 5 year period from October 2000 through September 2005 , 38 infant admitted to pediatric surgical unit at Tanta university hospital with intermediate anorectal malformation to undergo colostomy operation (4) .Each type of stoma has its own list of possible complications based upon its specific location and function ${ }^{(5)}$. Complications specific to stoma surgery stoma necrosis , retraction, stenosis, prolapse, mucocutaneous separation, peritonitis , skin complications (6) . the most commonly used definition for evidence based practice is, "the conscientious, explicit, and judicious use of the current best evidence in making decisions about the care of individual patients" (7). The most important reasons for consistently implementing evidence based nursing practice (EBNP) are that :it leads to the highest quality of care and the best patient outcomes . Implementing evidence based nursing practice (EBNP) is potentially 
beneficial for patients and healthcare systems, and for nurses . It can help nurses by facilitating informed and evidencebased clinical decision-making, helping them to keep updated with technologies, and enabling greater efficiency . In addition, it reduces healthcare costs and geographic variation in the delivery of care

. Findings from studies also indicate that clinicians report feeling more empowered and satisfied in their roles when they engage in evidence based practice EBP " ( 8) . Stoma nurse is a nurse who is practising in the field of stomal therapy but is uncertificated. Ostomy nurse coordinate or provide care and support to move the patient toward independent ostomy management .Ostomy nurse are in ideal position to initial assessments ,early interventions , patient $\backslash$ caregiver education, staff education, policy and procedure development, case management of complex cases ,cases quality improvement ${ }^{(9)}$. Evidence based practice for stoma care focus on three areas of care: pre-operative, post-operative, and followup. All children with ostomies, across the continuum of care, including the needs of the family and caregiver ${ }^{(\mathbf{1 0})}$.

\section{Aim of the study}

The aim of the study was to evaluate evidence based nurses' practice for children undergoing abdominal stoma outcome

\section{Research hypothesis}

The quality of nursing care delivered to children undergoing abdominal stoma expected to be improved after implementation of evidence based practice.

\section{Materials and method}

\section{Research design}

A quasi experimental research design was utilized to conduct this study .

\section{Setting}

The study was conducted at Pediatric Surgical Department Tanta University Hospital.

\section{Subjects}

All available nurses (40 nurses) who working at previously mentioned setting and providing direct care for children undergoing abdominal stoma operation.

A purposive sample of 40 children admitted to Pediatric Surgical Unit undergoing abdominal stoma operation and divided into two equal groups.

\section{Inclusion criteria of pediatric age group}

Age from 12 to 18 year, both sexes, Don't have any chronic diseases as diabetes mellitus, hypertension as it interfere with the wound stoma healing. 


\section{Tools of data collection}

Two tools were developed in order to obtain the necessary data for the study. Tool (I):A structured Questionnaire Schedule : it was developed by the researcher after reviewing the related literature to assess socio demographic data of nurses and children, nurses knowledge about the stoma care. it included two parts :

Part (I): a- Socio demographic data of the nurses which include

Age, educational level, residence, years of experience, and attendance to any conference or previously training related to stoma operation.

b-socio demographic data of the child such as age, sex, diagnosis and child's presenting problem, history of child's problem, surgical plan including type of ostomy (gastrostomy , ileostomy, urostomy, colostomy, jujnostmy); and expected duration(temporary, permanent).location of stoma .

Part (II): Nurses' knowledge regarding to stoma care. It was developed by the researcher after reviewing of literature to assess the nurses' knowledge about stoma care such as :Definition of stoma, types , indication of stoma operation for children Pre-operative care, Immediate Postoperative care, Stoma and peristomal skin care, Complication associated to stoma , Health education to parents about stoma care at home.

\section{Scoring system}

Two was given for correct and complete answer, one was given for correct and incomplete answer, zero for Incorrect or don't know answer. Number of questions was 51 question and total score was 102 . The Total score of knowledge items was calculated and score of more than $70 \%$ considered good level of knowledge , 60$70 \%$ fair level of knowledge \& less than $60 \%$ considered poor level of knowledge .

Tool (II): Nurses performance regarding stoma care and stoma, peristoma skin observation checklist : This tool was developed to observe nurses' performance related to stoma care in children and condition of stoma and peristomal skin. It included two parts :

Part (I) : Nurses performance observation checklist :This tool was developed by researcher to observe the quality of nursing care provided for children undergoing stoma operation. The researcher observed the nurses while demonstrating stoma care. It included Preoperative procedures, Immediate post operative care, Routine care of :fecal and urinary diversions procedures as pouch care and care of gastrostomy, and 
jejunostomy procedures, enteral feeding procedures. Peristoomal skin care procedures . Hygiene (bathing /clothing), exercises.

\section{Scoring system}

Two was given for done correctly and complete step, one for correct but incomplete step and for done incorrect or not done step zero score was given. Total steps of all procedures were 123 step with total score 246. Total score of nurses' performance items calculated and score of more than $70 \%$ considered good level of performance , 60-70\% fair level of performance \& less than $60 \%$ will considered poor level of performance.

\section{Part (II): Ostomy Skin Tool assessment}

Scoring system It is a standardized assessment tool developed by global group of experts in collaboration with an ostomy product company (2010).To evaluate and monitor the condition of stoma and peristomal skin reliably and accurately. Stoma assessment based on clinical observation of three domains color, appearance, protrusion . - Peristomal skin assessment based on clinical observation of three domains: discoloration , erosion/ulceration, and tissue overgrowth.

\section{Scoring system}

it is a likert type scale ranged from 0-3 where 0 represented normal stoma and peristoma skin and 3 represented the worst combination of severity and extent. the total score was 9 . whereas (< 4) represented mild complication, and $(<$ 7) represented moderate complication ,while (8and more) represented severe complication .

\section{Statistical Design}

The collected data were organized, categorized tabulated and analyzed using the Statistical Package for Social Sciences (SPSS). Data were presented in tables and charts using numbers, percentages, means، standard deviations and $t$ - test. Level of significance was threshold at 0.05 .

\section{Method}

Content validity and reliability :Tools of the study were tested for content validity by experts in the field of pediatrics, modifications were carried out accordingly.

\section{Ethical consideration : Nurses' consent} to participate in the study was obtained after explanation the aim of the study . Nurses were informed about the confidentiality of information obtained from them and the nature of the study . Data collected was confidential and used only for the purpose of the study. the study subjects were informed that they could with draw from the study at any time. 
Pilot study : It was conducted on $10 \%$ of nurses and children to test the tools for the clarity, visibility, and applicability of the study tools Then the necessary modification was done. The pilot study was excluded from the study sample

\section{Phases of the study}

The study was executed on three phases

\section{Assessment phase}

Interview with nurses was done at morning shifts .The nurses' knowledge and performance and children were assessed before and immediately and three month later from implementation of evidence based practice for Children Undergoing Abdominal Stoma.

\section{Implementation phase}

A purposive sample of children divided into two equal groups each group included 20 child by using tool II (part II). First group of children (control group) assessed before nurses' training, the second group (study group ) assessed immediately after nurses' training and three months later, to assess the effect of evidence based practice on the quality of care delivered to children on the condition of stoma and peristomal skin condition.

Preparation of suitable media for teaching the nurses as : lectures, PowerPoint, video , notes book were done.

Demonstration of procedures demonstrated by using real supplies of falnges, different types of pouches ,tube, cotton, scissors and gloves

Nurses were divided into 4 groups, ten nurses in each group .

\section{Evaluation phase}

The nurses and children were evaluated to determine the effectiveness program practice pre evidence based practice , immediately post and after three months later .

\section{Results}

Table 1 :

Frequency distribution of socio demographic characteristics of nurses showed that more than two thirds of nurses $(67.5 \%)$ their age more than 40 years and Mean \pm SD (41.125 \pm 8.979$)$. All of the nurses $100.00 \%$ were married, more than three quarter of nurses $(77.5 \%)$ had diploma degree. more than two thirds $(67.5 \%)$ of nurses have experience more than 20 year . unfortunately, the majority of nurses $(97.5 \%)$ didn't take any training courses about stoma .

Table (2)

Frequency distribution of socio demographic characteristics of children showed that $(45 \%)$ aged sixteen to less than eighteen years, compared to $(35 \%)$ of studied group after EBP at the same age , meanwhile, the majority of two groups 
studied before and after EBP ( $80.0 \%$, $90.0 \%$ respectively ) were males . the main cause of stoma is pathological as it

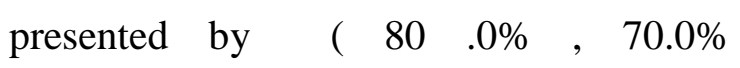
respectively ) . all of children studied after $\operatorname{EBP}(100.00 \%)$ have temporary stomas .

\section{Table (3)}

Percentage distribution of nurses' levels of knowledge pre, immediate, and three months post evidence based knowledge . Regarding to information of nurses about stomas and care of child before operation , the percentages of nurses $(72.5 \%, 60.0$ $\%$ respectively ) had poor level of knowledge before evidence based knowledge compared to , good level presented by $(100.0 \% \quad, 92.0 \%$ respectively ) immediately post guidelines. In relation to daily care of stoma and peristomal skin, the majority of nurses $(87.5 \%)$ had poor level before evidence based knowledge, while most of them $(95.0 \%, 72.5 \%$ respectively $)$ good level immediately post and after three months of EBK. Meanwhile, information of nurses about stomas complication and health education for child and parents before discharge, poor level of knowledge presented by $(37.0 \%, 27.0 \%$ respectively ) before evidence based knowledge , while good level presented by the majority of them $(95.0 \%, 92.5$ respectively ) immediately post and after three months .

\section{Table 4}

Illustrates percent distribution of total nurses' knowledge score pre, immediate, and three months post evidence based knowledge, it was observed that the majority of nurses $(97.5 \%)$ ) had poor total knowledge score pre evidence based knowledge .while all of them $(100.0 \%)$ had total good scores immediately post evidence based knowledge . the same table showed that total nurses' knowledge scores decreased 3 months after evidence based knowledge ( $90.0 \%$ ) .

\section{Table 5}

Shows the percentage distribution of nurses' levels of performance pre , immediate, and three months post evidence based practice . it was found that stoma site marking procedure presented by $(0.00 \%, 90.0 \%, 70.0 \%$ respectively $)$ at good level pre, immediately post and after three month evidence based practice while, care of jujnostomy or gastrostomy tube, it was found that, all nurses $(100.0 \%)$ at poor level at all procedures, in contrary to , gastrostomy or jejunpstomy tube removal, gastrostomy tube insertion, intermittent or bolus enteral feeding presented by $(100.0 \%)$ at 
good level immediately post evidence based practice.

\section{Table 6}

Shows the percentage distribution of nurses' total performance score pre , immediate, and three months post evidence based practice ,this table show that all nurses $(100.0 \%)$ had poor total performance score pre evidence based knowledge .while less than three quarter of them $(72.5 \%)$ had good total knowledge scores immediately post evidence based knowledge and the percentage of total nurses' performance scores decreased 3 months after evidence based knowledge as good level presented by $(60.0 \%)$.

\section{Table (7)}

Illustrates correlation between the total practice and knowledge total score pre , immediately post, and after three months of evidence based practice, it is noticed that , there was significant correlation between total knowledge score with total practice score pre evidence based practice with $\mathrm{p}$ value $=<0.001^{*}$, and immediately post EBP with $\mathrm{p}$ value $=<0.029^{*}$, and after three months with $\mathrm{p}$ value $=<0.046^{*}$. this means whenever total knowledge score increases, total practice score increased . The same table show, there is significant correlation between total practice score and total knowledge score with $\mathrm{p}$ value $=<0.001^{*}$.

\section{Table (8)}

Shows the correlation between severity of condition of stoma and peristomal skin pre , and post evidence based practice. It was found that , statistical significant difference between condition of stomas and peristomal skin pre and post evidence based practice with $\mathrm{p}$ value $=0.001 *$ this means that, condition of stomas and peristomal skin improved after nurses practiced evidence based practice 
Table 1: Percentage distribution of the studied nurses according to their socio demographic characteristics .

\begin{tabular}{|c|c|c|}
\hline \multirow{2}{*}{ Socio demographic Characteristics } & \multicolumn{2}{|c|}{$(\mathrm{N}=40)$} \\
\hline & No & $\%$ \\
\hline \multicolumn{3}{|l|}{ Age } \\
\hline$-20->30$ & 8 & 20.0 \\
\hline$-\quad 30->40$ & 5 & 12.5 \\
\hline$->40$ and more & 27 & 67.5 \\
\hline \multicolumn{3}{|l|}{ Mean \pm SD $=41.125 \pm 8.979$} \\
\hline \multicolumn{3}{|l|}{ Levels of education } \\
\hline -Diploma degree & 31 & 77.5 \\
\hline - Nursing technician Institute degree & 8 & 20.0 \\
\hline - Baccalaureate degree & 1 & 2.5 \\
\hline \multicolumn{3}{|l|}{ Marital status } \\
\hline -Married & 40 & 100.00 \\
\hline \multicolumn{3}{|l|}{ Years of experience } \\
\hline$-5-<10$ & 8 & 20.00 \\
\hline$-10-<20$ & 5 & 12.5 \\
\hline$->20$ & 27 & 67.5 \\
\hline \multicolumn{3}{|l|}{ Mean \pm SD $=21.475 \pm 8.964$} \\
\hline $\begin{array}{l}\text { Attendance to any training courses about } \\
\text { stoma }\end{array}$ & 1 & 2.5 \\
\hline Yes & 39 & 97.5 \\
\hline No & & \\
\hline
\end{tabular}


Table 2 : Percentage distribution of studied children according to their socio demographic characteristics

\begin{tabular}{|c|c|c|c|c|c|c|}
\hline \multirow[t]{2}{*}{$\begin{array}{c}\text { Socio Demographic } \\
\text { Characteristics }\end{array}$} & \multicolumn{2}{|c|}{$\begin{array}{c}\text { Control group } \\
\quad N=20\end{array}$} & \multicolumn{2}{|c|}{$\begin{array}{c}\text { Study group } \\
\mathrm{N}=20\end{array}$} & \multicolumn{2}{|c|}{ Chi-Square } \\
\hline & $\mathbf{N}$ & $\%$ & $\mathbf{N}$ & $\%$ & $\mathbf{X}^{2}$ & P-value \\
\hline \multicolumn{7}{|l|}{ Age } \\
\hline $12-<14 y$ & 5 & 25.00 & 6 & 30.00 & & \\
\hline $14-<16 y$ & 6 & 30.00 & 7 & 35.00 & 0.418 & 0.811 \\
\hline $16-<18 y$ & 9 & 45.00 & 7 & 35.00 & & \\
\hline \multicolumn{7}{|l|}{ Sex } \\
\hline Male & 16 & 80.00 & 18 & 90.00 & 0.784 & 0.376 \\
\hline Female & 4 & 20.00 & 2 & 10.00 & & \\
\hline \multicolumn{7}{|l|}{ Causes } \\
\hline Traumatic & 16 & 80.00 & 14 & 70.00 & & \\
\hline Pathological & 4 & 20.00 & 6 & 30.00 & 0.533 & 0.465 \\
\hline \multicolumn{7}{|l|}{ Site } \\
\hline Colostomy & 16 & 80.00 & 15 & 75.00 & & \\
\hline Ileostomy & 3 & 15.00 & 5 & 25.00 & 1.532 & 0.465 \\
\hline Urostomy & 1 & 5.00 & 0 & 0.00 & & \\
\hline Gastrostomy & 0 & 0.00 & 0 & 0.00 & & \\
\hline Jujnostomy & 0 & 0.00 & 0 & 0.00 & & \\
\hline \multicolumn{7}{|l|}{ Type } \\
\hline Permanent & 2 & 10.00 & 0 & 0.00 & 2.105 & 0.147 \\
\hline Temporary & 18 & 90.00 & 20 & 100.00 & & \\
\hline \multicolumn{7}{|l|}{ Location } \\
\hline Right & 14 & 70.00 & 17 & 85.00 & 1.290 & 0.256 \\
\hline Left & 6 & 30.00 & 3 & 15.00 & & \\
\hline
\end{tabular}


Table 3 : Percentage distribution of nurses' levels of knowledge pre, immediate, and three months post evidence based knowledge.

\begin{tabular}{|c|c|c|c|c|c|c|c|c|}
\hline \multirow{2}{*}{$\begin{array}{l}\text { Nurses levels of } \\
\text { knowledge }\end{array}$} & \multicolumn{2}{|c|}{ Before } & \multicolumn{2}{|c|}{$\begin{array}{c}\text { Immediate } \\
\text { after }\end{array}$} & \multicolumn{2}{|c|}{$\begin{array}{l}\text { After } 3 \\
\text { months }\end{array}$} & \multicolumn{2}{|c|}{ Chi-Square } \\
\hline & $\mathbf{N}$ & $\%$ & $\mathbf{N}$ & $\%$ & $\mathbf{N}$ & $\%$ & $\overline{X^{2}}$ & P-value \\
\hline $\begin{array}{l}\text { Information of nurses } \\
\text { about stomas :- } \\
\text { Good level } \\
\text { Fair level } \\
\text { Poor level }\end{array}$ & $\begin{array}{c}6 \\
10 \\
24\end{array}$ & $\begin{array}{l}15.00 \\
25.00 \\
60.00\end{array}$ & $\begin{array}{c}40 \\
0 \\
0\end{array}$ & $\begin{array}{c}100.00 \\
0.00 \\
0.00\end{array}$ & $\begin{array}{c}39 \\
1 \\
9\end{array}$ & $\begin{array}{c}97.50 \\
2.50 \\
0.00\end{array}$ & 90.969 & $<0.001 *$ \\
\hline $\begin{array}{l}\text { Care of child before the } \\
\text { operation:- } \\
\text { Good level } \\
\text { Fair level } \\
\text { Poor level }\end{array}$ & $\begin{array}{c}3 \\
8 \\
29 \\
\end{array}$ & $\begin{array}{c}7.50 \\
20.00 \\
72.50 \\
\end{array}$ & $\begin{array}{c}40 \\
0 \\
0 \\
\end{array}$ & $\begin{array}{c}100.00 \\
0.00 \\
0.00\end{array}$ & $\begin{array}{c}31 \\
7 \\
2 \\
\end{array}$ & $\begin{array}{c}77.50 \\
17.50 \\
2.50 \\
\end{array}$ & 85.519 & $<0.001 *$ \\
\hline $\begin{array}{l}\text { Care of child } \\
\text { immediately after the } \\
\text { operation:- } \\
\text { Good level } \\
\text { Fair level } \\
\text { Poor level }\end{array}$ & $\begin{array}{c}5 \\
12 \\
23\end{array}$ & $\begin{array}{l}12.50 \\
30.00 \\
57.50\end{array}$ & $\begin{array}{c}37 \\
3 \\
0\end{array}$ & $\begin{array}{c}92.50 \\
7.50 \\
0.00\end{array}$ & $\begin{array}{c}30 \\
9 \\
1\end{array}$ & $\begin{array}{c}75.00 \\
22.50 \\
2.50\end{array}$ & 71.083 & $<0.001^{*}$ \\
\hline $\begin{array}{l}\text { Daily care of stoma and } \\
\text { peristomal skin :- } \\
\text { Good level } \\
\text { Fair level } \\
\text { Poor level }\end{array}$ & $\begin{array}{c}0 \\
5 \\
35 \\
\end{array}$ & $\begin{array}{c}0.00 \\
12.50 \\
87.50 \\
\end{array}$ & $\begin{array}{c}38 \\
2 \\
0 \\
\end{array}$ & $\begin{array}{c}95.00 \\
5.00 \\
0.00 \\
\end{array}$ & $\begin{array}{c}29 \\
7 \\
4 \\
\end{array}$ & $\begin{array}{l}72.50 \\
17.50 \\
10.00 \\
\end{array}$ & 104.124 & $<0.001 *$ \\
\hline $\begin{array}{l}\text { Care of colostomy and } \\
\text { ileostomy and urostomy } \\
\text { bag } \\
\text { Good level } \\
\text { Fair level } \\
\text { Poor level }\end{array}$ & $\begin{array}{c}3 \\
6 \\
31\end{array}$ & $\begin{array}{c}7.50 \\
15.00 \\
77.50\end{array}$ & $\begin{array}{c}40 \\
0 \\
0\end{array}$ & $\begin{array}{c}100.00 \\
0.00 \\
0.00\end{array}$ & $\begin{array}{c}39 \\
0 \\
1\end{array}$ & $\begin{array}{c}97.50 \\
0.00 \\
2.50\end{array}$ & 102.700 & $<0.001 *$ \\
\hline $\begin{array}{l}\text { Care of gastrostomy or } \\
\text { jujnostomy tube :- } \\
\text { Good level } \\
\text { Fair level } \\
\text { Poor level }\end{array}$ & $\begin{array}{c}1 \\
2 \\
37 \\
\end{array}$ & $\begin{array}{c}2.50 \\
5.00 \\
92.50\end{array}$ & $\begin{array}{c}35 \\
4 \\
1\end{array}$ & $\begin{array}{c}87.50 \\
10.00 \\
2.50\end{array}$ & $\begin{array}{c}31 \\
6 \\
3 \\
\end{array}$ & $\begin{array}{c}77.50 \\
15.00 \\
7.50\end{array}$ & 92.828 & $<0.001 *$ \\
\hline $\begin{array}{l}\text { Information of nurses } \\
\text { about stomas } \\
\text { complication :- } \\
\text { Good level } \\
\text { Fair level } \\
\text { Poor level }\end{array}$ & $\begin{array}{l}12 \\
13 \\
15\end{array}$ & $\begin{array}{l}30.00 \\
32.50 \\
37.50\end{array}$ & $\begin{array}{c}38 \\
2 \\
0\end{array}$ & $\begin{array}{c}95.00 \\
5.00 \\
0.00\end{array}$ & $\begin{array}{c}31 \\
4 \\
5\end{array}$ & $\begin{array}{l}77.50 \\
10.00 \\
12.50\end{array}$ & 33.594 & $<0.001 *$ \\
\hline
\end{tabular}




\begin{tabular}{|l|c|c|c|c|c|c|c|c||}
\hline $\begin{array}{l}\text { Information of nurses } \\
\text { about health education } \\
\text { for child and parents } \\
\text { before discharge }\end{array}$ & & & & & & & & \\
Good level & 8 & 20.00 & 37 & 92.50 & 34 & 85.00 & 57.954 & $<0.001^{*}$ \\
Fair level & 21 & 52.50 & 3 & 7.50 & 5 & 12.50 & & \\
Poor level & 11 & 27.50 & 0 & 0.00 & 1 & 2.50 & & \\
\hline
\end{tabular}

Table 4 : Percentage distribution of nurses' total score of knowledge pre , immediately after, and three months post evidence based knowledge .

\begin{tabular}{|c|c|c|c|c|c|c|c|c|}
\hline \multirow{2}{*}{ Total knowledge score } & \multicolumn{2}{|c|}{ Before } & \multicolumn{2}{|c|}{$\begin{array}{c}\text { Immediate } \\
\text { after }\end{array}$} & \multicolumn{2}{|c|}{$\begin{array}{l}\text { After } 3 \\
\text { months }\end{array}$} & \multicolumn{2}{|c|}{ Chi-Square } \\
\hline & No & $\%$ & No & $\%$ & No & $\%$ & $\mathbf{X}^{2}$ & P-value \\
\hline $\begin{array}{l}\text { Good level } \\
\text { Fair level } \\
\text { Poor level }\end{array}$ & $\begin{array}{l}0 \\
1 \\
39\end{array}$ & $\begin{array}{l}0.00 \\
2.50 \\
97.50\end{array}$ & $\begin{array}{r}40 \\
0 \\
0\end{array}$ & $\begin{array}{r}100.00 \\
0.00 \\
0.00\end{array}$ & $\begin{array}{r}36 \\
2 \\
2\end{array}$ & $\begin{array}{r}90.00 \\
5.00 \\
5.00\end{array}$ & 110.901 & $<0.001 *$ \\
\hline
\end{tabular}

Table 5 : Percentage distribution of nurses' levels of performance pre, immediate, and three months post evidence based practice .

\begin{tabular}{|c|c|c|c|c|c|c|c|c|}
\hline \multirow[t]{2}{*}{ Nurses' levels of performance } & \multicolumn{2}{|c|}{ Before } & \multicolumn{2}{|c|}{$\begin{array}{c}\text { Immediate } \\
\text { after }\end{array}$} & \multicolumn{2}{|c|}{$\begin{array}{l}\text { After 3 } \\
\text { months }\end{array}$} & \multicolumn{2}{|c|}{ Chi-Square } \\
\hline & $\mathbf{N}$ & $\%$ & $\mathbf{N}$ & $\%$ & $\mathbf{N}$ & $\%$ & $\mathbf{X}^{2}$ & P-value \\
\hline $\begin{array}{l}\text { Pre operative care } \\
\text { Patient consent } \\
\text { Good level } \\
\text { Fair level } \\
\text { Poor level }\end{array}$ & $\begin{array}{c}0 \\
18 \\
22\end{array}$ & $\begin{array}{c}0.00 \\
45.00 \\
55.00\end{array}$ & $\begin{array}{c}24 \\
13 \\
3 \\
\end{array}$ & $\begin{array}{c}60.00 \\
32.50 \\
7.50\end{array}$ & $\begin{array}{l}19 \\
18 \\
3\end{array}$ & $\begin{array}{c}47.50 \\
45.00 \\
7.50\end{array}$ & 49.178 & $<0.001 *$ \\
\hline $\begin{array}{l}\text { Stoma site marking } \\
\text { Good level } \\
\text { Fair level } \\
\text { Poor level } \\
\end{array}$ & $\begin{array}{c}0 \\
0 \\
40\end{array}$ & $\begin{array}{c}0.00 \\
0.00 \\
100.00\end{array}$ & $\begin{array}{l}36 \\
4 \\
0\end{array}$ & $\begin{array}{c}90.00 \\
10.00 \\
0.00\end{array}$ & $\begin{array}{c}28 \\
6 \\
6\end{array}$ & $\begin{array}{l}70.00 \\
15.00 \\
15.00\end{array}$ & 99.796 & $<0.001 *$ \\
\hline $\begin{array}{l}\text { Lab values } \\
\text { Good level } \\
\text { Fair level } \\
\text { Poor level } \\
\end{array}$ & $\begin{array}{c}40 \\
0 \\
0\end{array}$ & $\begin{array}{c}100.00 \\
0.00 \\
0.00\end{array}$ & $\begin{array}{c}40 \\
0 \\
0\end{array}$ & $\begin{array}{c}100.00 \\
0.00 \\
0.00\end{array}$ & $\begin{array}{c}40 \\
0 \\
0\end{array}$ & $\begin{array}{c}100.00 \\
0.00 \\
0.00\end{array}$ & $\ldots$ & $\cdots$ \\
\hline $\begin{array}{l}\text { Day before surgery } \\
\text { Good level } \\
\text { Fair level } \\
\text { Poor level } \\
\end{array}$ & $\begin{array}{c}1 \\
26 \\
13\end{array}$ & $\begin{array}{c}2.50 \\
65.00 \\
32.50\end{array}$ & $\begin{array}{l}38 \\
2 \\
0\end{array}$ & $\begin{array}{l}95.00 \\
5.00 \\
0.00\end{array}$ & $\begin{array}{c}31 \\
7 \\
2\end{array}$ & $\begin{array}{c}77.50 \\
17.50 \\
5.00\end{array}$ & 80.200 & $<0.001 *$ \\
\hline $\begin{array}{l}\text { Immediate before surgery } \\
\text { Good level } \\
\text { Fair level }\end{array}$ & $\begin{array}{c}40 \\
0 \\
0\end{array}$ & $\begin{array}{c}100.00 \\
0.00 \\
0.00\end{array}$ & $\begin{array}{c}40 \\
0 \\
0\end{array}$ & $\begin{array}{c}100.00 \\
0.00 \\
0.00\end{array}$ & $\begin{array}{c}40 \\
0 \\
0\end{array}$ & $\begin{array}{c}100.00 \\
0.00 \\
0.00\end{array}$ & $\ldots \ldots$ & ..... \\
\hline
\end{tabular}




\begin{tabular}{|c|c|c|c|c|c|c|c|c|}
\hline Poor level & & & & & & & & \\
\hline $\begin{array}{l}\text { Immediate post operative care } \\
\text { Good level } \\
\text { Fair level } \\
\text { Poor level }\end{array}$ & $\begin{array}{c}8 \\
17 \\
15\end{array}$ & $\begin{array}{c}20.00 \\
42.50 \\
37.50\end{array}$ & $\begin{array}{c}40 \\
0 \\
0\end{array}$ & $\begin{array}{c}100.00 \\
0.00 \\
0.00\end{array}$ & $\begin{array}{c}34 \\
5 \\
1\end{array}$ & $\begin{array}{c}85.00 \\
12.50 \\
2.50\end{array}$ & 68.364 & $<0.001 *$ \\
\hline $\begin{array}{l}\text { Routine care of colostomy, } \\
\text { ileostomy, urostomy } \\
\text { child assessment } \\
\text { Good level } \\
\text { Fair level } \\
\text { Poor level }\end{array}$ & $\begin{array}{c}5 \\
5 \\
30\end{array}$ & $\begin{array}{l}12.50 \\
12.50 \\
75.00\end{array}$ & $\begin{array}{c}28 \\
9 \\
3\end{array}$ & $\begin{array}{c}70.00 \\
22.50 \\
7.50\end{array}$ & $\begin{array}{c}24 \\
11 \\
5\end{array}$ & $\begin{array}{l}60.00 \\
27.50 \\
12.50\end{array}$ & 53.872 & $<0.001 *$ \\
\hline $\begin{array}{l}\text { Prepare the pouch for application } \\
\text { Good level } \\
\text { Fair level } \\
\text { Poor level } \\
\end{array}$ & $\begin{array}{c}0 \\
1 \\
39\end{array}$ & $\begin{array}{c}0.00 \\
2.50 \\
97.50\end{array}$ & $\begin{array}{c}30 \\
5 \\
5\end{array}$ & $\begin{array}{l}75.00 \\
12.50 \\
12.50\end{array}$ & $\begin{array}{c}21 \\
11 \\
8\end{array}$ & $\begin{array}{l}52.50 \\
27.50 \\
20.00\end{array}$ & 77.708 & $<0.001 *$ \\
\hline $\begin{array}{l}\text { Application of the pouch } \\
\text { Good level } \\
\text { Fair level } \\
\text { Poor level } \\
\end{array}$ & $\begin{array}{c}24 \\
0 \\
16\end{array}$ & $\begin{array}{c}60.00 \\
0.00 \\
40.00\end{array}$ & $\begin{array}{c}40 \\
0 \\
0\end{array}$ & $\begin{array}{c}100.00 \\
0.00 \\
0.00\end{array}$ & $\begin{array}{c}39 \\
1 \\
0\end{array}$ & $\begin{array}{c}97.50 \\
2.50 \\
0.00\end{array}$ & 33.033 & $<0.001 *$ \\
\hline $\begin{array}{l}\text { Emptying clean pouch } \\
\text { Good level } \\
\text { Fair level } \\
\text { Poor level }\end{array}$ & $\begin{array}{c}6 \\
22 \\
12\end{array}$ & $\begin{array}{l}15.00 \\
55.00 \\
30.00\end{array}$ & $\begin{array}{c}40 \\
0 \\
0\end{array}$ & $\begin{array}{c}100.00 \\
0.00 \\
0.00\end{array}$ & $\begin{array}{c}40 \\
0 \\
0\end{array}$ & $\begin{array}{c}100.00 \\
0.00 \\
0.00\end{array}$ & 73.043 & $<0.001 *$ \\
\hline $\begin{array}{l}\text { Care jujnostomy or gastrostomy } \\
\text { tube } \\
\text { Child assessment and preparation } \\
\text { Good level } \\
\text { Fair level } \\
\text { Poor level }\end{array}$ & $\begin{array}{c}0 \\
0 \\
40\end{array}$ & $\begin{array}{c}0.00 \\
0.00 \\
100.00\end{array}$ & $\begin{array}{c}36 \\
3 \\
1\end{array}$ & $\begin{array}{c}90.00 \\
7.50 \\
2.50\end{array}$ & $\begin{array}{c}28 \\
4 \\
8\end{array}$ & $\begin{array}{l}70.00 \\
10.00 \\
20.00\end{array}$ & 90.153 & $<0.001 *$ \\
\hline $\begin{array}{l}\text { Skin care with dressing } \\
\text { change of jujnostomy or } \\
\text { gastrostomy tube } \\
\text { Good level } \\
\text { Fair level } \\
\text { Poor level } \\
\end{array}$ & $\begin{array}{c}0 \\
0 \\
40\end{array}$ & $\begin{array}{c}0.00 \\
0.00 \\
100.00\end{array}$ & $\begin{array}{c}39 \\
0 \\
1\end{array}$ & $\begin{array}{l}97.50 \\
0.00 \\
2.50\end{array}$ & $\begin{array}{c}32 \\
8 \\
0\end{array}$ & $\begin{array}{c}80.00 \\
20.00 \\
0.00\end{array}$ & 128.682 & $<0.001 *$ \\
\hline $\begin{array}{l}\text { Gastrostomy or jejunpstomy } \\
\text { tube removal } \\
\text { Good level } \\
\text { Fair level } \\
\text { Poor level }\end{array}$ & $\begin{array}{c}0 \\
0 \\
40\end{array}$ & $\begin{array}{c}0.00 \\
0.00 \\
100.00\end{array}$ & $\begin{array}{c}40 \\
0 \\
0\end{array}$ & $\begin{array}{c}100.00 \\
0.00 \\
0.00\end{array}$ & $\begin{array}{c}33 \\
5 \\
2\end{array}$ & $\begin{array}{c}82.50 \\
12.50 \\
5.00\end{array}$ & 120.078 & $<0.001 *$ \\
\hline $\begin{array}{l}\text { Gastrostomy tube insertion } \\
\text { Good level } \\
\text { Fair level } \\
\text { Poor level }\end{array}$ & $\begin{array}{c}0 \\
0 \\
40\end{array}$ & $\begin{array}{c}0.00 \\
0.00 \\
100.00\end{array}$ & $\begin{array}{c}40 \\
0 \\
0\end{array}$ & $\begin{array}{c}100.00 \\
0.00 \\
0.00\end{array}$ & $\begin{array}{c}38 \\
2 \\
0\end{array}$ & $\begin{array}{c}95.00 \\
5.00 \\
0.00\end{array}$ & 123.077 & $<0.001 *$ \\
\hline $\begin{array}{l}\text { Intermittent or bolus enteral } \\
\text { feeding } \\
\text { Good level } \\
\text { Fair level } \\
\text { Poor level }\end{array}$ & $\begin{array}{c}0 \\
0 \\
40\end{array}$ & $\begin{array}{c}0.00 \\
0.00 \\
100.00\end{array}$ & $\begin{array}{c}40 \\
0 \\
0\end{array}$ & $\begin{array}{c}100.00 \\
0.00 \\
0.00\end{array}$ & $\begin{array}{c}38 \\
2 \\
0\end{array}$ & $\begin{array}{c}95.00 \\
5.00 \\
0.00\end{array}$ & 123.077 & $<0.001 *$ \\
\hline
\end{tabular}


Table 6 : percentage distribution of Total score of nurses' performance pre , immediate , and three months post evidence based practice .

\begin{tabular}{|c|c|c|c|c|c|c|c|c|}
\hline \multirow{2}{*}{$\begin{array}{c}\text { Total score of nurses' } \\
\text { Performance }\end{array}$} & \multicolumn{2}{|c|}{$\begin{array}{l}\text { Before } \\
\text { EBP }\end{array}$} & \multicolumn{2}{|c|}{$\begin{array}{l}\text { Immediate } \\
\text { after EBP }\end{array}$} & \multicolumn{2}{|c|}{$\begin{array}{c}\text { After } 3 \\
\text { months } \\
\text { EBP }\end{array}$} & \multicolumn{2}{|c|}{ Chi-Square } \\
\hline & $\mathbf{N}$ & $\%$ & $\mathbf{N}$ & $\%$ & $\mathbf{N}$ & $\%$ & $\mathbf{X}^{2}$ & P-value \\
\hline $\begin{array}{l}\text { Good level } \\
\text { Fair level } \\
\text { Poor level }\end{array}$ & $\begin{array}{c}0 \\
0 \\
40\end{array}$ & $\begin{array}{c}0.00 \\
0.00 \\
100.00\end{array}$ & $\begin{array}{c}29 \\
8 \\
3\end{array}$ & $\begin{array}{c}72.50 \\
20.00 \\
7.50\end{array}$ & $\begin{array}{c}24 \\
10 \\
6\end{array}$ & $\begin{array}{l}60.00 \\
25.00 \\
15.00\end{array}$ & 98.005 & $<0.001 *$ \\
\hline
\end{tabular}

Table 7 : Correlation between the total practice and knowledge total score pre , immediately post , and after three months of evidence based practice .

\begin{tabular}{|l|l|l|}
\hline \multirow{2}{*}{ Total practice and knowledge total score } & \multicolumn{2}{l|}{ Total knowledge score \% } \\
\cline { 2 - 3 } & R & P-value \\
\hline Before EBP & 0.132 & $<0.001^{*}$ \\
\hline Immediately after EBP & 0.027 & $<0.029^{*}$ \\
\hline After 3 months EBP & -0.098 & $<0.046^{*}$ \\
\hline Total practice score \% & 0.893 & $<0.001^{*}$ \\
\hline
\end{tabular}

Table 8 : Correlation between severity of condition of stoma and peristomal skin pre, and post evidence based practice.

\begin{tabular}{|l|c|c|c|c|c|c|}
\hline \multirow{2}{*}{$\begin{array}{l}\text { Stoma and peristomal } \\
\text { skin }\end{array}$} & \multicolumn{2}{|l|}{ Pre EBP } & \multicolumn{2}{l|}{ Post EBP } & \multicolumn{2}{l|}{ Chi-Square } \\
\cline { 2 - 6 } & N=20 & \% & N=20 & \% & $\mathbf{X}^{\mathbf{2}}$ & P-value \\
\hline Mild degree & 3 & 15.00 & 14 & 70.00 & & \\
\cline { 1 - 5 } & 12 & 60.00 & 6 & 30.00 & \multirow{2}{*}{14.118} & \multirow{2}{*}{$0.001^{*}$} \\
\hline Moderate degree & 5 & 25.00 & 0 & 0.00 & & \\
\hline Severe degree & 20 & 100.00 & 20 & 100.00 & & \\
\hline
\end{tabular}




\section{Tanta Scientific Nursing Journal}

\section{Discussion}

Formation of a stoma can result in physiological, psychological and emotional problems for the child, young person and their family (Hurber 2001) ${ }^{(\mathbf{1 1})}$ .The current study aimed to evaluate evidence based nurses' practice for children undergoing abdominal stoma outcome.in the present study, Concerning nurses' years of experience and educational level, this study showed that, nurses with more 20 years represented by more than two thirds and more than three quarter of nurses had diploma degree . This result was in agreement with Abd El Salam (2006) ${ }^{(12)}$ who conducted study to assist The effect of nurses' training on the care of abdominal stoma patients and found that nurses with more than 10 years of experience represented by less than one third and diploma of nurses represented by less than two thirds. In relation to previous training courses, it was found that all nurses except one nurses hadn't attend any previous training courses about stoma care . this result was in contrary to Abd El Salam (2006) (12) who found that, slightly less than half of nurses had previous training. On the same context noticeable findings of the study was that more than half of the patients were male.

This is supported by Baldwin (2009) ${ }^{(13)}$ who reported that, More than half patients undergoing colostomy were males . Considering nurses' knowledge about stomas, the results of this study showed that, there was improvement in nurses knowledge immediately post and after three months .this result is supported by Bhzeh (2013) ${ }^{(14)}$ who revealed that all of nurses had knowledge about the standards regarding colostomy at an unsatisfactory level. In relation to total performance score of nurses . this study revealed that all nurses at poor level pre evidence based practice . this result in congruent with Bhzeh (2013) ${ }^{(14)}$ who showed that total practice level regarding the nursing care standards of colostomy was at unsatisfactory level. Meanwhile the study clarify that the improvement of performance levels less than improvement of knowledge levels immediately post and after three months of evidence based practice . it can be attributed to shortage of nursing staff, lack of organization support . this result in congruent with Caroline (2009) ${ }^{(15)}$ Organizational barriers (lack of time and lack of nursing autonomy) were the top perceived barriers for application of evidence based practice and 
Facilitators were learning opportunities, culture building, and availability and simplicity of resources. Concerning occurrence of the stomal and peristomal complication, present study revealed that the highest percentages of complications were for discoloration of peristomal skin and lacerated stomas in control group. these result were in agreement with Herlufsen (2006) ${ }^{(16)}$ reported a cross sectional study of 202 persons with permanent ileostomies,colostomies and urostomies, that more than half of patients had mild complications, one third of patients had moderate complications, and severe complications presented by only one tenth. Types of skin damage included erosion, maceration, erythema and irritant dermatitis. Collectively, these accounted for more than three quarters of all complications. Peristomal skin disorders persisted for more than three months in three quarters of patients. This study revealed that statistical significant difference between condition of stomas and peristomal skin pre and post evidence based practice ..this result in agreement Rafii, F, Naseh L (2013) ${ }^{\text {(2) }}$ mentioned that, In stoma care self-efficacy subscale, the highest obtained mean score were: preventing stoma bleeding and damage ,follow the stoma therapist's instructions for handling the stoma and found that the findings of this study can be useful to enhance nurses' knowledge to improve training and supportive interventions for stoma patients and their families

\section{Conclusion}

It can be concluded that, the educational guidelines and training program were effective regarding improvement of nurses' knowledge and performance regarding stomas conditions .

\section{Recommendations}

Continuing periodically in-service training programs should be introduced to the nurses on regular basis by different methods and materials of teaching.

Written booklets and pictures should be provided to illustrate the stoma care for both nurses and patients.

Researches are needed about guidelines to increase competences of children and their parents of stoma care.

\section{References}

1-Martins L, Ayello E, Claessens I, Steen H, Hentze P, Sibbald R. Jemec G. The ostomy skin tool: tracking peristomal skin changes. British Journal of Nursing $2010 ; 19(15): 960,632$ - 4.

2-Rafii, F L, Naseh L. Parvizy, P. Selfefficacy and its dimensions in patients with intestinal Stoma. Iran Journal of Nursing $2013 ; 26$ (83):10- 8. 
3- Gundogan K, Yurci A , Coskun R, Baskol M, S Gursoy . Outcomes of percutaneous endoscopic gastrostomy in hospitalized patients at a tertiary care center in Turkey . European Journal of Clinical Nutrition 2014 ; 68 (4): 437440.

4-El Halaby E, El Safoury H, Hashish A . Primary repair of high and intermediate anorectal malformation in the neonatal period. Annals Of Pediatric Surgery 2006; 2(1) : 53-76.

5- Doughty D . Ostomy care: the added considerations for cancer patients, The Journal of Supportive Oncology 2005 ; $3: 71-72$.

6- Shabbir J, Britton D. Stoma complications: a literature overview.

Colorectal Dis. 2010 ; 12(10):958-64.

7- Ellen EO, Mazurek B Melny . Evidence Based Practice In Nursing \& Healthcare : A Guide To Best Practice, 2nd ed . USA Lippincott Williams \& Wilkins Co., 2011.

8- Reigle, B S, Stevens K R., Belcher J V., Huth, M M .. Evidence-based practice and the road to Magnet status. Journal of Nursing Administration 2008 ; 38(2) : 97-102.

9- Moore S. Nurse perceptions of ostomy patients \& their ostomy care competence: Journal of Home Care Provider 2004 ; 3 (4): 214-220.

10-Waller $\mathrm{M}$. Pediatric stoma care nursing in the UK and Irland . British Journal of Nursing $2008 ; 17$ (1) : 25- 9.
11- Hurber E . Caring for patient with a stoma, living with stoma : what impossible become possible . kranken sonis infirm 2001 ; 94(4) : 66-70.

12- Abd-Salam R . The effect of nurses' training on the care of abdominal stoma patients . PHD , Egypt : Tanta University 2006.

13- Baldwin C, Grant $\mathrm{M}$, Wendel $\mathrm{C}$, Hornbrook M, Herrinton L, McMullen $\mathrm{C}$, Krouse R . Gender differences in sleep disruption and fatigue on quality of life among persons with ostomies . Journal of Holistic Nursing 2009 ; 5 (4):335-43.

14- Bhzeh N M, Teleb S M, Mahmoud M A , Soliman A M.Colostomy: Developing Nursing Care Standards for Patient with Colostomy Med. Journal of Cairo University 2013;81(2):57-64.

15- Caroline E B , Mary A W, Laurie E. Nursing practice, knowledge, attitudes and perceived barriers to evidencebased practice at an academic medical center of Advanced Nursing . Glaser Journal 2009 ; 65( 2) : 371-81.

16- Herlufsen P, Olsen A G , Carlsen B , Nybaek H, Karlsmark T, Laursen, T N .Study of peristomal skin disorders in patients with permanent stomas. British Journal of Nursing 2006; 15(16) : 854-862. 